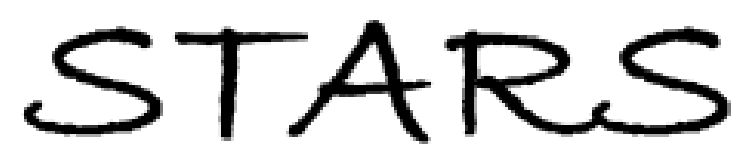

University of Central Florida

STARS

Faculty Bibliography 2000s

Faculty Bibliography

$1-1-2009$

\title{
Albedos of Small Jovian Trojans
}

Yanga R. Fernández

University of Central Florida

David Jewitt

Julie E. Ziffer

Find similar works at: https://stars.library.ucf.edu/facultybib2000

University of Central Florida Libraries http://library.ucf.edu

This Article is brought to you for free and open access by the Faculty Bibliography at STARS. It has been accepted for inclusion in Faculty Bibliography 2000s by an authorized administrator of STARS. For more information, please contact STARS@ucf.edu.

\section{Recommended Citation}

Fernández, Yanga R.; Jewitt, David; and Ziffer, Julie E., "Albedos of Small Jovian Trojans" (2009). Faculty Bibliography 2000s. 1525.

https://stars.library.ucf.edu/facultybib2000/1525

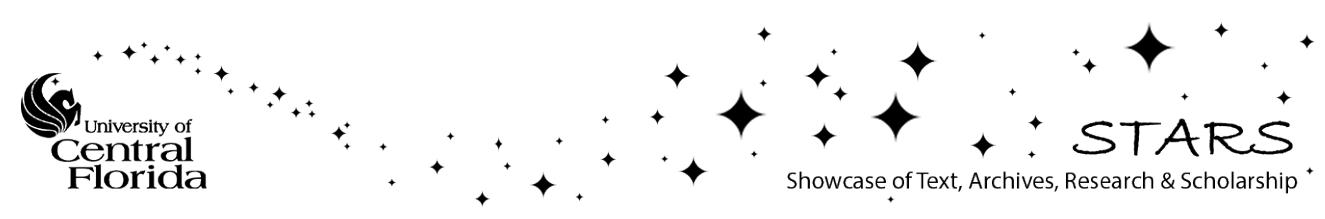




\title{
ALBEDOS OF SMALL JOVIAN TROJANS
}

\author{
YANGa R. FernÁNDEZ ${ }^{1}$, DAVID JewitT ${ }^{2}$, AND Julie E. ZIFFER ${ }^{3}$ \\ ${ }^{1}$ Department of Physics, University of Central Florida, 4000 Central Florida Boulevard, Orlando, FL 32816-2385, USA \\ ${ }^{2}$ Institute for Astronomy, University of Hawaii, 2680 Woodlawn Drive, Honolulu, HI 96822, USA \\ ${ }^{3}$ Department of Physics, University of Southern Maine, 96 Falmouth Street, Portland, ME 04104-9300, USA \\ Received 2008 October 12; accepted 2009 May 5; published 2009 June 8
}

\begin{abstract}
We present thermal observations of 44 Jovian Trojan asteroids with diameters $D$ ranging from 5 to $24 \mathrm{~km}$. All objects were observed at a wavelength of $24 \mu \mathrm{m}$ with the Spitzer Space Telescope. Measurements of the thermal emission and of scattered optical light, mostly from the University of Hawaii $2.2 \mathrm{~m}$ Telescope, together allow us to constrain the diameter and geometric albedo of each body. We find that the median $R$-band albedo of these small Jovian Trojans is about 0.12 , much higher than that of "large" Trojans with $D>57 \mathrm{~km}(0.04)$. Also the range of albedos among the small Trojans is wider. The small Trojans' higher albedos are also glaringly different from those of cometary nuclei, which match our sample Trojans in diameter, however, they roughly match the spread of albedos among (much larger) Centaurs and trans-Neptunian objects. We attribute the Trojan albedos to an evolutionary effect: the small Trojans are more likely to be collisional fragments and so their surfaces would be younger. A younger surface means less cumulative exposure to the space environment, which suggests that their surfaces would not be as dark as those of the large, primordial Trojans. In support of this hypothesis is a statistically significant correlation of higher albedo with smaller diameter in our sample alone and in a sample that includes the larger Trojans. This correlation of albedo and radius implies that the true size distribution of small Trojans is shallower than the visible magnitude distribution alone would suggest, and that there are approximately half the Trojans with $D>1 \mathrm{~km}$ than previously estimated.
\end{abstract}

Key words: infrared: solar system - minor planets, asteroids

Online-only material: machine-readable and VO tables

\section{INTRODUCTION}

Jupiter's Trojan asteroids inhabit two swarms centered on the L4 and L5 Lagrangian points located 5.2 AU from the Sun and from the planet. More than 2700 Trojans are known at the time of writing. Based on optical studies, the total population larger than $1 \mathrm{~km}$ in radius has been estimated by various workers: Jewitt et al. (2000) estimated $\sim 1.6 \times 10^{5}$ such objects in the L4 swarm; Szabó et al. (2007) estimated $\sim 2.4 \times 10^{5}$ in both swarms combined; Yoshida \& Nakamura (2005) estimated $\sim 2.4 \times 10^{5}$ in the L4 swarm; and Nakamura \& Yoshida (2008) estimated $\sim 0.63 \times 10^{5}$ in the L4 swarm and $\sim 0.34 \times 10^{5}$ in the L5. The magnitude-derived size distribution resembles a broken power law (Jewitt et al. 2000), and is such that the bulk of the mass (approximately $10^{-4} M_{\oplus}$, where $M_{\oplus}=6 \times 10^{24} \mathrm{~kg}$ is the mass of the Earth) is contained within the largest objects. By number and by mass, the Trojan population is only slightly inferior to the population of the main-belt asteroids. However, the observational attention given to the Trojans so far is miniscule compared to that lavished on the main-belt objects, and many of the basic properties of Jupiter's Trojans remain poorly known. The Trojans have been reviewed alongside the irregular satellites of Jupiter, to which they may be closely related, by Jewitt et al. (2004) and separately by Dotto et al. (2008).

Scientific interest in the Trojans focuses both on their origin and on their composition. How and when they were trapped in 1:1 mean-motion resonance with Jupiter remains unknown. Capture at a very early epoch in association with planet formation and capture much later, in a dynamical clearing phase in the solar system, are both under current consideration (Morbidelli et al. 2005; Marzari \& Scholl 2007). The snow-line in the solar system was most likely inside the orbit of Jupiter (Garaud \& Lin 2007), so if they formed in situ or at a more distant location in the Sun's protoplanetary disk, the Trojans could have incorporated water as bulk ice. In this sense, the Trojans might share as much in common with the nuclei of comets as with the classical, rocky asteroids. Observationally, the measured Trojans resemble the nuclei of short-period comets in their optical colors (Jewitt \& Luu 1990; Fornasier et al. 2007) and albedos (Fernández et al. 2003, Paper I), tending to reinforce by association the possibility that they might be cometlike, ice-rich bodies. On the other hand, low-resolution spectral observations in the near-infrared (near-IR) have uniformly failed to reveal absorption bands that could be attributed to water ice or, indeed, to show any absorption bands at all (Luu et al. 1994; Dumas et al. 1998; Emery \& Brown 2003; Yang \& Jewitt 2007). The low albedos, neutral to reddish optical colors and featureless near-IR spectra are compatible with, but not uniquely diagnostic of, irradiated, complex organics (Cruikshank et al. 2001).

The absence of water ice is easily understood as a consequence of sublimation, even at Jupiter's distance. For example, dirty (absorbing) water ice exposed at the subsolar point on a Trojan at 5.2 AU sublimates in equilibrium at a rate of $\dot{m} \sim 8 \times 10^{-7} \mathrm{~kg} \mathrm{~m}^{-2} \mathrm{~s}^{-1}$, corresponding to recession of the sublimating surface at speed $\dot{m} / \rho \sim 2 \mathrm{~cm} \mathrm{yr}^{-1}$, where $\rho \sim$ $10^{3} \mathrm{~kg} \mathrm{~m}^{-3}$ is the bulk density. In a few years, any exposed dirty water ice on a Jovian Trojan would recede into the surface by a depth greater than the diurnal thermal skin depth (i.e., approximately $5-10 \mathrm{~cm}$ on a body rotating with a period $\sim 0.5$ day and a thermal diffusivity $\kappa \sim 10^{-7} \mathrm{~m}^{2} \mathrm{~s}^{-1}$ ). Clean (i.e., pure) surface ice could survive much longer, by virtue of its higher albedo and lower temperature, but sustaining clean surface ice will be difficult in the face of micrometeorite gardening and contamination. Just as with the nuclei of comets, then, the Trojans could have ice-rich interiors but relatively (or even, completely) ice-free surfaces composed of refractory, particulate matter (mantles). 
Table 1

Target List and Observing Circumstances

\begin{tabular}{|c|c|c|c|c|c|c|c|c|c|}
\hline No. & Name & $\mathrm{Ln}$ & $\begin{array}{c}H \\
(\mathrm{mag})\end{array}$ & Tel. & $\begin{array}{c}\text { UT Date } \\
\text { (yyyy-mm-dd) }\end{array}$ & $\begin{array}{c}\text { UT } \\
\text { (at start) }\end{array}$ & $\begin{array}{c}r \\
(\mathrm{AU})\end{array}$ & $\begin{array}{c}\Delta \\
(\mathrm{AU})\end{array}$ & $\begin{array}{c}\alpha \\
\left({ }^{\circ}\right)\end{array}$ \\
\hline$(58153)$ & $1988 \mathrm{RH}_{11}$ & L5 & 13.2 & $\mathrm{~S}$ & 2004-11-04 & $01: 29: 25$ & 5.706 & 5.506 & 10.2 \\
\hline " & " & " & " & $\mathrm{H}$ & 2005-04-07 & 07:06:09 & 5.749 & 5.627 & 10.0 \\
\hline ” & $"$ & " & " & $\mathrm{H}$ & 2005-04-08 & $07: 44: 34$ & 5.749 & 5.643 & 10.0 \\
\hline (37572) & $1989 \mathrm{UC}_{5}$ & L5 & 13.4 & $\mathrm{~S}$ & 2004-11-10 & $08: 55: 22$ & 5.431 & 5.368 & 10.8 \\
\hline ” & " & ", & " & $\mathrm{H}$ & 2005-04-07 & $07: 11: 15$ & 5.575 & 5.226 & 10.0 \\
\hline " & " & " & $"$ & $\mathrm{H}$ & 2005-04-08 & $07: 49: 39$ & 5.576 & 5.242 & 10.0 \\
\hline$(58366)$ & $1995 \mathrm{OD}_{8}$ & L4 & 13.7 & $\mathrm{~S}$ & 2005-04-08 & $22: 17: 04$ & 5.483 & 5.297 & 10.5 \\
\hline ” & " & " & " & $\mathrm{H}$ & $2005-06-30$ & $07: 31: 15$ & 5.473 & 4.499 & 3.4 \\
\hline " & " & " & $"$ & $\mathrm{H}$ & $2005-06-30$ & 09:04:49 & 5.473 & 4.499 & 3.4 \\
\hline
\end{tabular}

Notes. Here $\mathrm{L} n$ indicates the swarm (Lagrange point) in which each object resides; $H$ gives the absolute magnitude as listed by the Minor Planet Center at URL http://cfa-www.harvard.edu/iau/lists/JupiterTrojans.html; the "Tel." column lists which telescope was used: "S" = Spitzer Space Telescope, "H" = University of Hawaii $2.2 \mathrm{~m}$ Telescope; $r, \Delta$, and $\alpha$ list the heliocentric distance, geocentric/Spitzercentric distance, and geocentric/ Spitzercentric phase angle as given by JPL's Horizons system.

(This table is available in its entirety in machine-readable and Virtual Observatory (VO) forms in the online journal. A portion is shown here for guidance regarding its form and content.)

In this case, it is possible that collisions within each swarm (Marzari et al. 2002) occasionally cause previously embedded and relatively pristine material to be exposed to space. While no water ice has been definitively detected spectroscopically on the surfaces of larger Trojans (as mentioned above), smaller bodies, currently just beyond the range of ground-based spectroscopic observation, may hold some remnant near-surface ice.

In order to address these topics, we are investigating some of the physical properties of the known Trojans. In earlier work (Paper I), we found that the geometric albedos of Trojans larger than $\sim 60 \mathrm{~km}$ in diameter ("large" Trojans) are uniform. The mean $R$-band geometric albedo of such objects is $0.044 \pm 0.002$ and the standard deviation is just 0.008 . (These are transformed from the paper's $V$-band results using the average color derived by Fornasier et al. (2007) of $V-R=0.45$.) In our sample of 32 objects, there was only one outlier (4709 Ennomos), with an albedo of $0.14 \pm 0.02$. We interpreted this uniformity in reflectance to be indicative of mostly similar evolutionary history across the large end of the Trojan distribution. Not all such Trojans are exactly the same-as shown, for example, in the distribution of visible- and near-IR spectral slopes (Fornasier et al. 2007) — but the narrow spread in albedos lies in stark contrast to other outer solar system populations such as Centaurs and trans-Neptunian objects (Stansberry et al. 2008). Interestingly, the albedo of large Trojans most closely matches the cometary nuclei (Lamy et al. 2004) even though there is a large size mismatch.

In this paper, we report results from our program to study the albedos of "small" Trojans that more closely match the comets in diameter. In Section 2 we present our observations, in Section 3 we discuss the interpretation, and in Section 4 we discuss some implications of our work.

\section{OBSERVATIONS}

We have two data sets, one obtained with the Spitzer Space Telescope (Werner et al. 2004) that provided us with mid-IR imaging, and another with the University of Hawaii $2.2 \mathrm{~m}$ Telescope that provided us with visible-wavelength imaging. Table 1 provides a list of our targets and the circumstances of the observations. The targets were chosen to have excellent ephemerides so that there would be no doubt about the success of the Spitzer observations. At the time we prepared the project, our targets were among the smallest numbered Trojans known (as judged by $H$, the absolute magnitude).

\subsection{Spitzer Data}

We used the Multiband Imaging Photometry for Spitzer (MIPS; Rieke et al. 2004) aboard Spitzer to observe all 44 small Trojans during Cycle 1. Each Trojan was observed in "photometry" mode using the $24 \mu \mathrm{m}$ imager (effective wavelength $\lambda=23.68 \mu \mathrm{m}$ ), a 128-by-128 array of Si:As impurity band conduction detectors. The scale is 2.55 arcsec per pixel, and the spatial resolution is diffraction-limited (Rayleigh criterion of 7.1 arcsec). The integration time was $132 \mathrm{~s}$, using $3 \mathrm{~s}$ exposure times and 3 cycles, resulting in 44 individual raw exposures. Each visit to a Trojan lasted 6.7 minutes, including observing overheads. Raw data were processed by the Spitzer pipeline version 14.4.0 to produce flux-calibrated "BCD" (basic calibrated data) images. A discussion of the pipeline processing is given by Gordon et al. (2005). In general, the data quality was high and the Trojans provided good signal-to-noise ratios in the individual frames. No latents or streaks were seen.

To measure the flux density of each Trojan, we used two independent methods. First, we used MOPEX (Makovoz \& Marleau 2005) to obtain photometry of an object using its individual BCD images. The targets were bright enough that stacking to boost the signal was not necessary. This gave us 44 separate samples of a Trojan's brightness, with which we could calculate an appropriate mean and error, and also readily identify bad frames. Second, we used Interactive Data Language (IDL) software to analyze post-BCD mosaics provided by the Spitzer pipeline. These post-BCD data are combinations of the BCD images and have had array distortions rectified (Spitzer Science Center 2008).

In both methods, aperture photometry was performed usually using an aperture of radius 3.0 BCD pixels ( $7.65 \mathrm{arcsec})$, though reduced to 2.0 or $2.5 \mathrm{BCD}$ pixels when the Trojan was near a background object. The results were compared and in all cases the differences were at the few percent level. Averages and propagated errors were then calculated.

This photometry was then corrected for aperture loss and for color to produce a final measurement of the monochromatic 
Table 2

Photometry

\begin{tabular}{|c|c|c|c|c|c|}
\hline No. & Name & Tel. & $\begin{array}{c}\text { UT Date } \\
\text { (yyyy-mm-dd) }\end{array}$ & $\begin{array}{c}\text { UT } \\
\text { (at start) }\end{array}$ & $\begin{array}{c}F \text { or } m_{R} \\
(\mathrm{mJy} \text { or } \mathrm{mag})\end{array}$ \\
\hline$(58153)$ & $1988 \mathrm{RH}_{11}$ & $\mathrm{~S}$ & 2004-11-04 & $01: 29: 25$ & $13.54 \pm 0.16$ \\
\hline$"$ & " & $\mathrm{H}$ & 2005-04-07 & 07:06:09 & $20.472 \pm 0.075$ \\
\hline$”$ & " & $\mathrm{H}$ & 2005-04-08 & $07: 44: 34$ & $20.567 \pm 0.072$ \\
\hline (37572) & $1989 \mathrm{UC}_{5}$ & S & 2004-11-10 & $08: 55: 22$ & $5.14 \pm 0.06$ \\
\hline$"$ & " & $\mathrm{H}$ & 2005-04-07 & $07: 11: 15$ & $20.725 \pm 0.074$ \\
\hline$"$ & ” & $\mathrm{H}$ & 2005-04-08 & $07: 49: 39$ & $20.955 \pm 0.079$ \\
\hline$(58366)$ & $1995 \mathrm{OD}_{8}$ & $\mathrm{~S}$ & 2005-04-08 & $22: 17: 04$ & $6.37 \pm 0.28$ \\
\hline , & $"$ & $\mathrm{H}$ & $2005-06-30$ & $07: 31: 15$ & $20.475 \pm 0.023$ \\
\hline , & " & $\mathrm{H}$ & $2005-06-30$ & 09:04:49 & $20.788 \pm 0.298$ \\
\hline
\end{tabular}

Notes. Here the "Tel." column lists which telescope was used: "S" = Spitzer Space Telescope, "H" = University of Hawaii $2.2 \mathrm{~m}$ Telescope; the " $F$ or $m_{R}$ " column lists either the flux density $F$ at a wavelength of $23.68 \mu \mathrm{m}$ as observed by Spitzer or the Cousins $R$ magnitude $m_{R}$ as observed by the UH $2.2 \mathrm{~m}$ Telescope.

(This table is available in its entirety in machine-readable and Virtual Observatory (VO) forms in the online journal. A portion is shown here for guidance regarding its form and content.)

flux density. All Trojans appeared as point sources in all images, facilitating an aperture correction. Color corrections were calculated from the shape of the expected spectral energy distribution that results from the thermal model (see Section 3) and the known Trojan-Spitzer-Sun angles and distances.

Our photometry is listed in Table 2, with $1 \sigma$ error bars. Errors in the photometry result from uncertainty in the photon counting, in measuring an appropriate sky background, and in the repeatability of the photometry from BCD to BCD.

\subsection{UH 2.2 m Telescope Data}

Optical photometry was obtained on the nights of UT 2005 April 7, April 8, June 28, June 29, and June 30 using the University of Hawaii $2.2 \mathrm{~m}$ Telescope located atop Mauna Kea, Hawaii. We used a Tektronix charge-coupled device (CCD) camera located at the $f / 10$ Cassegrain focus to image the Trojans through an $R$-band filter approximating the KronCousins photometric system. Image scale with this setup was 0.219 arcsec per pixel. The image quality delivered by the telescope, including the effects of the atmosphere and windshake of the telescope, was typically $0.8-1.0$ arcsec full width at half-maximum (FWHM).

Photometric calibration was obtained using observations of standard stars from the list by Landolt (1992), giving us effectively Cousins $R$-band magnitudes. We selected the faintest standard stars and those having broadband colors most similar to the Sun in order to minimize photometric uncertainties owing to the shutter and to color terms introduced by the use of broadband filters. We also observed the standards at airmasses similar to the airmasses of the Trojans, to minimize atmospheric extinction corrections. The sky on all nights was photometric except for part of the night of April 7, as judged by the real-time data from the "Skyprobe" instrument on the Canada-France-Hawaii Telescope. Data taken through thin clouds on April 7 were calibrated using the photometry of the same field stars observed on April 8.

Photometry was performed using concentric, circular projected apertures, typically from 4 to 7 pixels $(0.9-1.5$ arcsec) in radius. Several of our targets were observed at low Galactic latitude and so we took care to select an aperture size and sky location so as to exclude flux from background stars. Integration times employed were short enough that trailing of the Trojans relative to the fixed stars was comparable to, or less than, the image FWHM, so resulting in no photometric consequence.

Aperture and color corrections were applied to our photometry and the resulting final Cousins $R$-band magnitudes are listed in Table 2 , with $1 \sigma$ error bars. Note that for 12 of our 44 objects, optical data were not obtained or were unusable due to stellar crowding. Error in the photometry results mainly from uncertainty in the aperture correction and in the determination of an appropriate sky background.

\section{PHYSICAL PARAMETERS}

\subsection{Thermal Model}

The basic radiometric method to obtain an effective diameter $D$ and geometric albedo $p$ is to solve two equations with these two unknowns, first done many years ago (Allen 1970; Matson 1971; Morrison 1973) and described in detail by (e.g.) Lebofsky $\&$ Spencer (1989). One must observe the reflected sunlight (usually in visible wavelengths) and the thermal emission (usually in mid-IR wavelengths); the former is proportional to $D^{2} p$, while the latter is proportional to $D^{2}(1-p q)$, where $q$ is the phase integral. In our study, we observed Trojans only in Cousins $R$-band, so the geometric albedo is specific to that band and represented by $p_{R}$.

The method requires knowing the distribution of temperature across the object's surface, which itself depends on many parameters including the orientation and magnitude of the spin vector and the thermal diffusivity/thermal inertia of the surface materials. The spin vectors and thermal properties of the sample Trojans are unfortunately unknown. The median rotation period for main-belt asteroids of the appropriate diameter scale is about $6 \mathrm{hr}$ (Pravec et al. 2002). Thermal inertias of primitive asteroids are less well studied, but recent work on cometary nuclei and Centaurs suggest that their thermal inertias are roughly $\sim 10 \mathrm{~J}$ $\mathrm{m}^{-2} \mathrm{~K}^{-1} \mathrm{~s}^{-1 / 2}$ (e.g., Fernández et al. 2006; Groussin et al. 2007; Li et al. 2007; Lamy et al. 2008; Groussin et al. 2009). These parameters, if applicable to small Trojans, indicate that at $5 \mathrm{AU}$ the Trojans would lie in the "slow rotator" regime (Spencer et al. 1989).

The thermal model that we have employed to interpret our data is the "NEA Thermal Model" (NEATM) devised by Harris (1998), a simple and widely used modification to the older "standard thermal model" (STM; Lebofsky \& Spencer 1989). The STM and NEATM generally apply if the rotation is so slow or the thermal inertia so low that every point on the surface is near instantaneous equilibrium with the impinging solar radiation. In the case of zero thermal inertia, the temperature is maximum at the subsolar point and decreases as $(\cos \vartheta)^{1 / 4}$, where $\vartheta$ is the local solar zenith angle.

To use NEATM we must make some assumptions. We assume that emissivity $\epsilon=0.9$ and the phase slope parameter $G=0.05$. We also assume a value for the beaming parameter, $\eta$, which is a rough proxy for thermal inertia and the effects of surface roughness, nightside emission, and beaming from, e.g., craters. In Paper I, we found that $\eta=0.94$ was an appropriate average value for the large Trojans, so we employ that value again here. We note that recent work (e.g., Harris \& Davies 1999; Delbó et al. 2003, 2007) indicates that small bodies can have a variety of values for $\eta$, and that the beaming parameter is often strongly dependent on the phase angle. Fortunately, all of our sample objects were observed at similar low phase angles. We address in Section 4 the effect that changing $\eta$ would have on our results. 


\subsection{Nonsimultaneity}

Technically the derivation of diameter and albedo from this method requires that the observations be done simultaneously, or at least while knowing the rotational context of the observations. Neither condition was satisfied by our data sets, since it is difficult to schedule ground-based observations to match Spitzer observations. This means that the diameters and albedos that we derive may not be exactly correct for a specific object. Depending on the different rotational phases at which the thermal and reflected signals are measured, the derived diameters and albedos could be either too high or too low by an amount that depends on the deviation of the shape from spheroidal.

Fortunately, this effect should average out. Our sample size is large enough, and we have detected all of our targets at significant signal-to-noise ratio so that we are not missing the faint end of the sample. Thus, we have an approximately equal number of Trojans with both too high and too low albedos. While the albedos for individual objects may be off from their true values, the average and median of the ensemble of apparent albedos should be close to the true average and median. The spread of the distribution will be wider than it really is, but the extent of this spread can be estimated (see Section 4).

In any case, to make use of the multiwavelength photometry we had to convert the visible magnitudes to account for the differing heliocentric distance $r$, geo/Spitzercentric distance $\Delta$, and geo/Spitzercentric phase angle $\alpha$. In other words, we needed to estimate what each Trojan's magnitude would be had it been observed by the UH $2.2 \mathrm{~m}$ Telescope at the same geometry at which it was observed by Spitzer. The correction to the measured magnitude is $5 \log \left(r_{\mathrm{i}} / r_{\mathrm{v}}\right)+5 \log \left(\Delta_{\mathrm{i}} / \Delta_{\mathrm{v}}\right)+\Phi\left(G, \alpha_{\mathrm{i}}\right)-\Phi\left(G, \alpha_{\mathrm{v}}\right)$, where subscripts "v" and "i" refer to the visible and IR observations, $\Phi$ is the phase function, and $G$ is the phase slope parameter.

For the 12 objects with no visible-wavelength data, we have used the absolute magnitude $H$ (as given by the Minor Planet Center $\left.(\mathrm{MPC})^{4}\right)$ and the average Trojan $V-R$ color $(0.45$; Fornasier et al. 2007) to predict what the visible magnitude would be. We assumed an uncertainty of $\pm 0.1 \mathrm{mag}$ for $H$.

\subsection{Modeling Results}

Since there are two measurements and two parameters to be fit, there are no degrees of freedom with which to use, say, a $\chi^{2}$ statistic. Therefore, we employed a Monte Carlo method with which to estimate the uncertainties of $D$ and $p_{R}$ based on the uncertainties in the photometry. For each pair of photometric points - one mid-IR and one visible-we created 500 pairs of hypothetical measurements distributed normally about the actual measured values and with sigmas equal to the actual error bars. We then derived the appropriate $D$ and $p_{R}$ that fits each pair, giving us 500 pairs of $D$ and $p_{R}$. The means and standard deviations of these distributions of $D$ and $p_{R}$ essentially became our "best-fit values" and "error bars."

For the 12 objects with no visible-wavelength data, we effectively have only the one visible data point derived from $H$. For the other 32 objects, however, there are multiple visible data points. For a Trojan with $N$ such visible measurements, we paired each measurement in turn with the single mid-IR measurement to create $N$ estimates for both $D$ and $p_{R}$ using the

\footnotetext{
4 http://cfa-www.harvard.edu/iau/lists/JupiterTrojans.html
}

Table 3

Physical Parameters and Formal Errors

\begin{tabular}{|c|c|c|c|}
\hline No. & Name & $D(\mathrm{~km})$ & $p_{R}$ \\
\hline$(58153)$ & $1988 \mathrm{RH}_{11}$ & $14.92 \pm 0.10$ & $0.074 \pm 0.005$ \\
\hline$(37572)$ & $1989 \mathrm{UC}_{5}$ & $8.62 \pm 0.06$ & $0.139 \pm 0.019$ \\
\hline$(58366)$ & $1995 \mathrm{OD}_{8}$ & $9.52 \pm 0.20$ & $0.108 \pm 0.020$ \\
\hline$(58475)$ & $1996 \mathrm{RE}_{11}$ & $12.22 \pm 0.10$ & $0.085 \pm 0.031$ \\
\hline (192393) & $1996 \mathrm{TT}_{22}$ & $7.80 \pm 0.06$ & $0.088 \pm 0.009$ \\
\hline (37789) & $1997 \mathrm{UL}_{16}$ & $14.22 \pm 0.22$ & $0.062 \pm 0.009$ \\
\hline$\ldots$ & $1998 \mathrm{WM}_{24}$ & $6.90 \pm 0.16$ & $0.094 \pm 0.009$ \\
\hline$\cdots$ & $1998 \mathrm{WO}_{39}$ & $10.20 \pm 0.12$ & $0.104 \pm 0.010$ \\
\hline$(40262)$ & $1999 \mathrm{CF}_{156}$ & $10.80 \pm 0.08$ & $0.079 \pm 0.007$ \\
\hline$(59355)$ & $1999 \mathrm{CL}_{153}$ & $9.10 \pm 0.08$ & $0.102 \pm 0.009$ \\
\hline$(60257)$ & $1999 \mathrm{WB}_{25}$ & $10.66 \pm 0.08$ & $0.155 \pm 0.056$ \\
\hline$(60322)$ & $1999 \mathrm{XB}_{257}$ & $24.02 \pm 0.14$ & $0.067 \pm 0.005$ \\
\hline (192942) & $2000 \mathrm{AB}_{219}$ & $7.72 \pm 0.04$ & $0.118 \pm 0.011$ \\
\hline (60388) & $2000 \mathrm{AY}_{217}$ & $11.12 \pm 0.08$ & $0.043 \pm 0.004$ \\
\hline (162396) & $2000 \mathrm{CV}_{120}$ & $13.90 \pm 0.08$ & $0.058 \pm 0.005$ \\
\hline$(60421)$ & $2000 \mathrm{CZ}_{31}$ & $12.98 \pm 0.08$ & $0.050 \pm 0.005$ \\
\hline$(62692)$ & $2000 \mathrm{TE}_{24}$ & $18.38 \pm 0.12$ & $0.073 \pm 0.005$ \\
\hline$(68112)$ & $2000 \mathrm{YC}_{143}$ & $10.38 \pm 0.08$ & $0.180 \pm 0.024$ \\
\hline (63193) & $2000 Y_{118}$ & $13.70 \pm 0.22$ & $0.094 \pm 0.017$ \\
\hline$(63259)$ & $2001 \mathrm{BS}_{81}$ & $9.96 \pm 0.08$ & $0.094 \pm 0.008$ \\
\hline$(88240)$ & $2001 \mathrm{CG}_{21}$ & $12.16 \pm 0.16$ & $0.108 \pm 0.018$ \\
\hline (63284) & $2001 \mathrm{DM}_{46}$ & $10.26 \pm 1.32$ & $0.252 \pm 0.050$ \\
\hline$(63279)$ & $2001 \mathrm{DW}_{34}$ & $8.32 \pm 0.58$ & $0.213 \pm 0.022$ \\
\hline$(28960)$ & $2001 \mathrm{DZ}_{81}$ & $14.52 \pm 0.18$ & $0.123 \pm 0.021$ \\
\hline (109266) & $2001 \mathrm{QL}_{110}$ & $9.22 \pm 0.06$ & $0.079 \pm 0.004$ \\
\hline$(156222)$ & $2001 \mathrm{UB}_{91}$ & $7.34 \pm 0.06$ & $0.120 \pm 0.008$ \\
\hline$(156250)$ & $2001 \mathrm{UM}_{198}$ & $9.04 \pm 0.06$ & $0.084 \pm 0.012$ \\
\hline$(64326)$ & $2001 \mathrm{UX}_{46}$ & $17.28 \pm 0.10$ & $0.066 \pm 0.002$ \\
\hline (158333) & $2001 \mathrm{WW}_{25}$ & $8.56 \pm 0.08$ & $0.205 \pm 0.040$ \\
\hline$\ldots$ & $2002 \mathrm{CG}_{205}$ & $7.64 \pm 0.14$ & $0.141 \pm 0.010$ \\
\hline$(43627)$ & $2002 \mathrm{CL}_{224}$ & $8.58 \pm 0.08$ & $0.205 \pm 0.016$ \\
\hline$(65179)$ & $2002 \mathrm{CN}_{224}$ & $10.60 \pm 0.02$ & $0.113 \pm 0.012$ \\
\hline (166115) & $2002 \mathrm{CO}_{208}$ & $6.66 \pm 0.06$ & $0.111 \pm 0.010$ \\
\hline$\cdots$ & $2002 \mathrm{CS}_{266}$ & $5.64 \pm 0.14$ & $0.260 \pm 0.037$ \\
\hline$(65174)$ & $2002 \mathrm{CW}_{207}$ & $11.78 \pm 0.08$ & $0.167 \pm 0.052$ \\
\hline$(65206)$ & $2002 \mathrm{DB}_{13}$ & $11.72 \pm 0.10$ & $0.127 \pm 0.038$ \\
\hline (89913) & $2002 \mathrm{EC}_{24}$ & $12.66 \pm 0.10$ & $0.113 \pm 0.005$ \\
\hline$(65211)$ & $2002 \mathrm{EK}_{1}$ & $7.20 \pm 0.28$ & $0.334 \pm 0.020$ \\
\hline (195258) & $2002 \mathrm{EN}_{52}$ & $12.00 \pm 0.06$ & $0.041 \pm 0.004$ \\
\hline$(65227)$ & $2002 \mathrm{ES}_{46}$ & $14.04 \pm 0.10$ & $0.179 \pm 0.003$ \\
\hline$(65217)$ & $2002 \mathrm{EY}_{16}$ & $12.70 \pm 0.08$ & $0.165 \pm 0.028$ \\
\hline$(65250)$ & $2002 \mathrm{FT}_{14}$ & $10.72 \pm 0.10$ & $0.074 \pm 0.007$ \\
\hline (183358) & $2002 \mathrm{VM}_{131}$ & $9.62 \pm 0.06$ & $0.108 \pm 0.006$ \\
\hline$(58096)$ & Oineus & $14.40 \pm 0.50$ & $0.088 \pm 0.013$ \\
\hline
\end{tabular}

Notes. Here $D$ is the effective diameter and $p_{R}$ is the Cousins $R$-band geometric albedo. For both quantities, the quoted error is a $1 \sigma$ formal error given the modeling assumptions and the photometric uncertainties.

Monte Carlo idea described above. We then averaged together all the estimates to create a single overall estimate of diameter and albedo. We also propagated the errors except in cases where the variance of either $D$ or $p_{R}$ among the $N$ estimates was significantly larger than the nominal error estimate, in which case we simply used the standard deviation itself.

Our final values of diameter and albedo are given in Table 3. The table includes all 32 objects with multiwavelength data, as well as the 12 with only IR data. It is important to note however that the table's values do not account for the nonsimultaneity of the IR and visible data, and that an individual radius and albedo may be off due to the lack of rotational context. The error bars, likewise, do not include any such systematic effects. We discuss this further in the following section. 


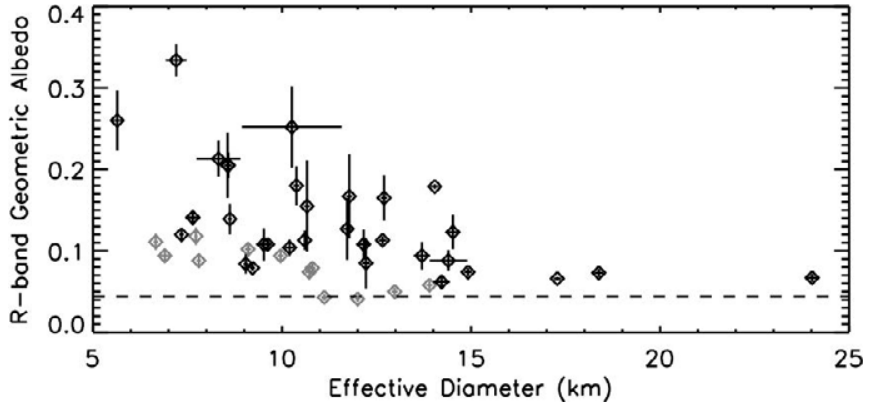

Figure 1. Scatter plot of the 44 albedos and diameters derived in this survey. The 12 objects for which we used $H$ are shown in gray; the 32 objects for which we have multiwavelength data are shown in black. The mean albedo of large Trojans as found by us (Paper I), and translated from $V$-band to $R$-band, is indicated with a horizontal dashed line. There is a correlation of albedo with radius among the black points that is significant at the $3.4 \sigma$ level.

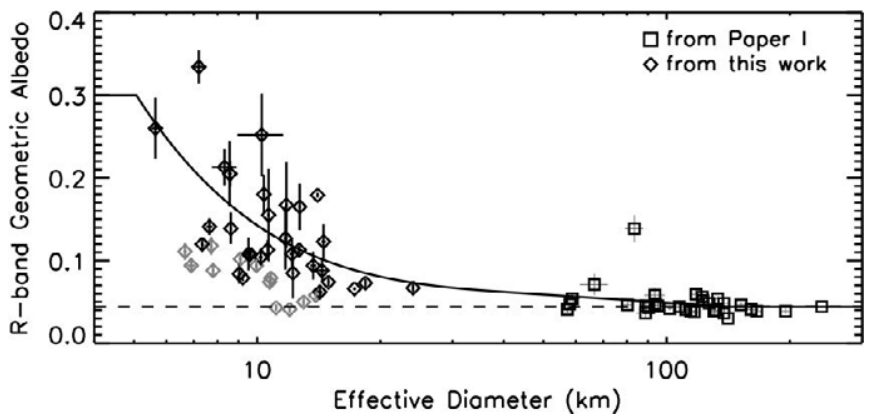

Figure 2. Combination of the radii and albedos from the current survey (diamonds) and from our earlier work (squares; Paper I). Diamond grayscale is the same as Figure 1. All 32 points from Paper I have been included here. Among the 64 black points there is a correlation of albedo with radius that is significant at the $6.8 \sigma$ level. Horizontal dashed line indicates the mean large-Trojan albedo of 0.044 . Solid piecewise curve represents an ad hoc function used to investigate the size distribution; see Section 4.5.

\section{DISCUSSION}

\subsection{Ensemble Properties and Correlations}

A plot of diameter versus albedo from Table 3 is shown in Figure 1. The most striking feature is the evidence of a trend where the smaller Trojans have higher albedos, or at least a higher likelihood of having higher albedos. The Spearman rankorder correlation coefficient among the diameters and albedos of these 44 objects is -0.493 , which corresponds to a probability of the two quantities being uncorrelated of only $6.7 \times 10^{-4}$. In terms of the sum-squared difference of the ranks the correlation is significant at the $3.2 \sigma$ level.

Since an absolute magnitude reported by the MPC (or by other agencies for that matter) could potentially be more uncertain than the $0.1 \mathrm{mag}$ we have arbitrarily assumed-owing to uncertainty in color transformations, in phase darkening laws, and in weighting schemes - we have also analyzed the statistical significance of the apparent trend in Figure 1, while excluding the 12 objects for which we did not have our own visible data. We believe this is a more robust analysis since it uses the results of more uniform data sets. In this case, the Spearman rankorder correlation coefficient is -0.610 , which corresponds to an even lower probability of noncorrelation of $2.1 \times 10^{-4}$. The significance of the sum-squared difference of the ranks is even higher, $3.4 \sigma$. So the trend is statistically significant regardless of whether we include 32 or 44 objects.

In Figure 2, we add the 32 albedos from our earlier work (Paper I) onto the same plot. Including these data with the best

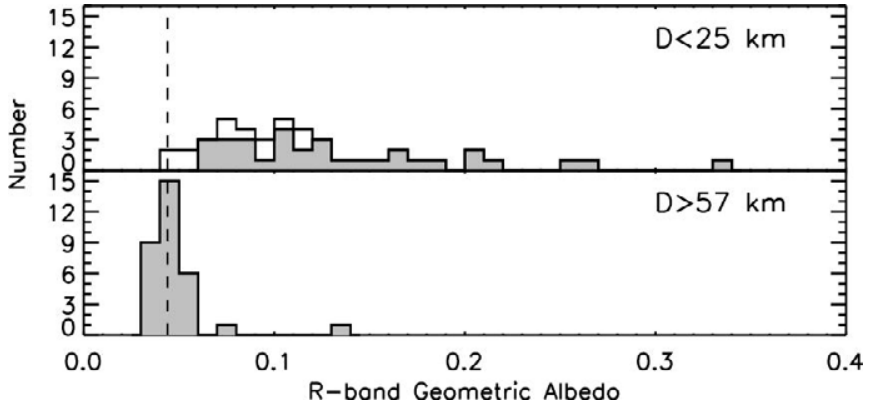

Figure 3. Histograms of Trojan albedos. Top panel: distribution of small Trojan albedos presented in this work. The 32 albedos from multiwavelength objects are shown with the filled histogram; including all 44 objects gives the unfilled histogram. Bottom panel: distribution of large Trojan albedos reported by us in Paper I. The means and shapes of the distributions are quite different.

32 gives us 64 data points, and the correlation appears even more pronounced. The Spearman rank-order correlation coefficient among the diameters and albedos of these 64 objects is -0.852 , which corresponds to a probability of the two quantities being uncorrelated of only $4.1 \times 10^{-19}$. In terms of the sum-squared difference of the ranks the correlation is significant at the $6.8 \sigma$ level.

It is clear from Figure 2 that the average albedo of a small Trojan is larger than that of a large Trojan. This is more readily demonstrated in Figure 3, where the histograms of the two populations are compared. We can also see that the range of albedos is larger. Note that our earlier work (Paper I) reported $V$-band albedos, so we have scaled those albedos to $R$-band by using the average Trojan color $V-R=0.45$ (Fornasier et al. 2007). To be quantitative, we compare the averages, medians, and standard deviations of the two populations in Table 4. (We have listed separately the values for our whole sample of 44 and those values for the 32 objects that have multiwavelength data.) Clearly, the typical small-Trojan albedo is higher than that of the large Trojans.

Table 4 and Figure 3 indicate that the range of albedos among small Trojans is wider than the range for the large Trojans, but we must be careful since the width of the distribution is at least partly artificial due to the lack of simultaneity in our data sets as described in Section 3. We can estimate how much of this spread is real based on a study of Trojan light curves by Mann et al. (2007). They observed 114 Trojans with sparse sampling and derived a distribution of photometric ranges. Looking at just their 26 Trojans with apparent diameters under $35 \mathrm{~km}$ (so as to approximately match the diameters of Trojans in our sample), their distribution has a broad peak with ranges $\Delta m$ from 0 to $0.3 \mathrm{mag}$. The distribution then tails off toward $\Delta m=0.8$ mag. The average range is $\Delta m=0.24 \mathrm{mag}$ and the median is $\Delta m=0.22 \mathrm{mag}$. From this we take $0.24 \mathrm{mag}$ to be the appropriate average $\Delta m$ for the Trojans in our sample. This means that an optical magnitude would be at most \pm 0.24 mag different from what it would have been had the observation been taken simultaneously with the Spitzer observation. The average offset would be somewhat less, approximately half of this, since it is unlikely that we would have observed each Trojan at a maximum in the light curve with one telescope and at a minimum with the other telescope. However, we leave the offset at 0.24 mag as a worst-case scenario, corresponding to a change in visible flux density by a factor of $10^{0.4 \times( \pm 0.24)}=0.80$ or 1.25 . To first order that would also be the factor change in the albedo. So, if hypothetically all the small Trojans had a true albedo of 
Table 4

Ensemble $R$-Band Geometric Albedos

\begin{tabular}{|c|c|c|c|c|c|c|}
\hline Group & $N$ & Average & Median & Std. Dev. & Source & Excluding \\
\hline "Small" & 44 & $0.121 \pm 0.003$ & $0.105 \pm 0.004$ & 0.062 & This work & None \\
\hline
\end{tabular}

Notes. Here "large" and "small" in the "Group" column refer to Trojans of diameter greater than 57 km and Trojans of diameter less than $24 \mathrm{~km}$, respectively; $N$ is the number of objects included in that row's calculations; and "Excluding" indicates which objects are excluded from that row's calculations. Error bars on the averages come from propagating errors of the albedos themselves; error bars on the medians come from Monte Carlo simulations.
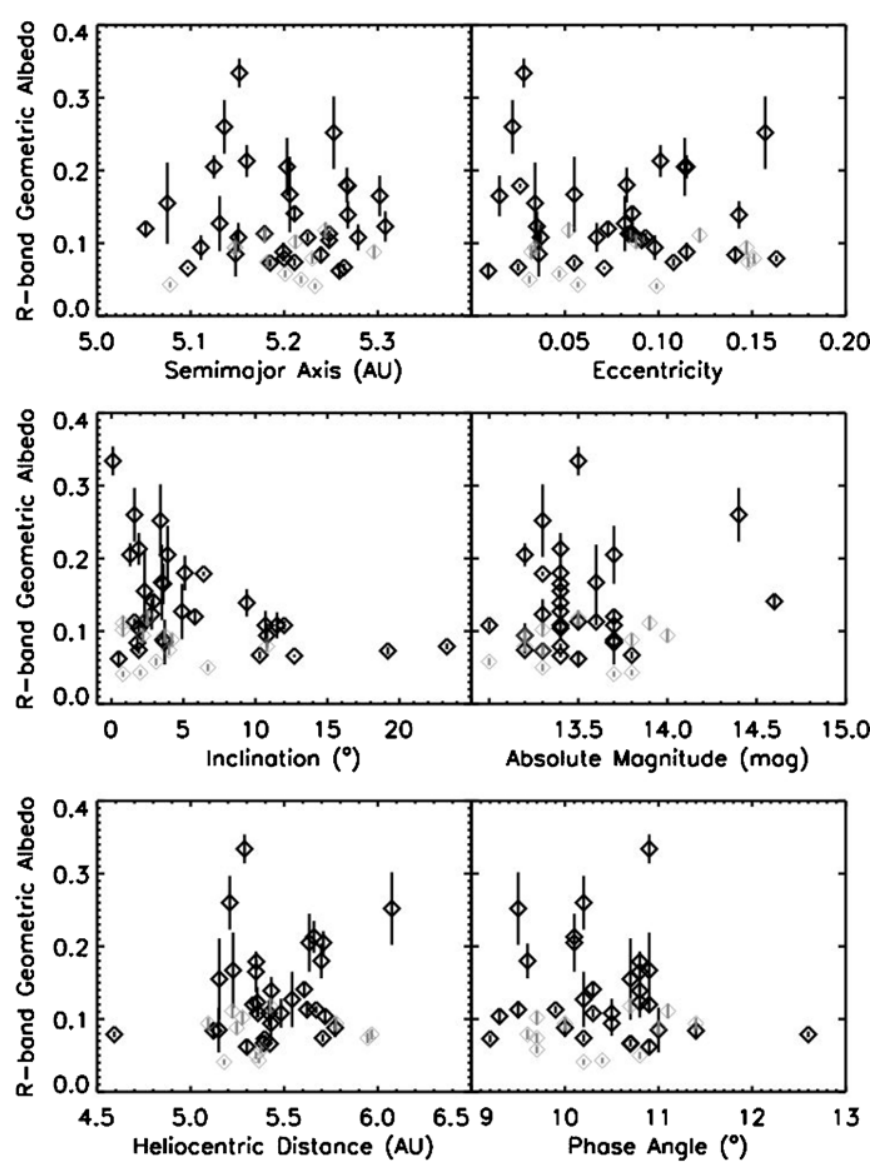

Figure 4. Scatter plots of the small Trojan albedos with various orbital and observed quantities. Diamond grayscale is the same as Figure 1. The only panel with some indication of a correlation is the inclination, but this is significant at only the $2 \sigma$ level.

exactly 0.100 , then we would expect to see a distribution that ranges from $(0.100 \times 0.80=) 0.080$ to $(0.10 \times 1.25=) 0.125$. Clearly, the histogram of small Trojan albedos is wider than this. In fact, the observed albedos from 0.04 to 0.12 could only be explained with an average $\Delta m$ of about $0.6 \mathrm{mag}$. So unless the small Trojans have substantially higher typical axial ratios than were measured by Mann et al. (2007), the spread of small Trojan albedos really is intrinsically wider than that of the large Trojans.

We searched for correlations between albedo and other properties of the small Trojans. These comparisons are shown in Figure 4, where we plotted albedos against three orbital parameters and three observed parameters. The only panel suggesting a correlation is the inclination, in which higher albedo Trojans are more likely to have low inclination. However, the Spearman rank-order correlation coefficient among the 32 multiwavelength objects is only -0.357 , which corresponds to a probability of the two quantities being uncorrelated of 0.045 . In terms of the sum-squared difference of the ranks, the correlation is significant at only the $2.0 \sigma$ level. Adding in the 32 largeTrojan albedos from Paper I improves the correlation, but this is likely to be spurious since the Trojans in the two surveys do not have overlapping inclinations.

\subsection{Discovery Bias}

It is important to consider whether the trend in Figure 2 is a product of discovery bias. That is, perhaps we are measuring higher albedos because such small Trojans are more likely to be discovered; after all, a Trojan of a given diameter with 0.12 albedo will be $1.09 \mathrm{mag}$ brighter than one with the same diameter but 0.044 albedo. If the high-albedo small Trojans are near the limit of what can be discovered by asteroid surveys, then 1.09 mag of difference would render a hypothetical low-albedo subpopulation invisible. On the other hand, the situation is not quite this simple since a Trojan could have been discovered at another lunation when it was brighter. Furthermore, the unknown rotational period and axial ratio make predicting when a Trojan can and cannot be discovered difficult.

A simple argument does suggest though that at least the fraction of high-albedo small Trojans (however one wants to define "high") is greater than that fraction among the large Trojans. Only 3\% (1/32) of the large Trojans from Paper I have albedos above the median albedo we have measured here, 0.117 . If only $3 \%$ of all small Trojans in reality have albedos above this value, then the asteroid surveys would have to have missed a vast population of Trojans with $5<D<25 \mathrm{~km}$, a population that is about 17 times larger than what has actually been discovered. This seems unlikely.

We can test this situation more rigorously however by assuming an overall albedo distribution to the small Trojans and then determining what the measured albedo distribution would be for the asteroids that are actually discovered by the asteroid surveys. To do this, we created a virtual population of small Trojans and assigned them absolute magnitudes $H$ such that the ensemble's distribution of $H$ matched that for the real Trojans as measured by Jewitt et al. (2000). They found that for Trojans below a diameter of about $50 \mathrm{~km}$, the cumulative magnitude distribution $N$ as a function of absolute magnitude $H$ is $N(<H) \propto 10^{\alpha H}$, with $\alpha=0.40 \pm 0.06$. For our modeling, we assumed $\alpha=0.40$ precisely. The number of objects in the simulation was 1.26 million.

We then created an albedo probability distribution $P(p)$ to dictate what $V$-band albedo each virtual object would be assigned. (We discuss $P$ further below.) From this we calculated the diameters $D$ for all virtual objects using $D=10^{-0.2 H} \times$ $1329 \mathrm{~km} / \sqrt{p}$. 
Next, we assigned orbits to all virtual objects using a fivedimensional distribution of Trojan semimajor axis $(a)$, orbital eccentricity $(e)$, orbital inclination $(i)$, argument of perihelion $(\omega)$, and longitude of ascending node $(\Omega)$. This five-dimensional distribution was derived empirically by extracting the orbital elements as compiled by the Minor Planet Center. ${ }^{5}$ For ease, we let each virtual object's perihelion time $t_{P}$ be randomly chosen between 1992 January 1 and 2004 January 1, i.e., sometime within a 12-year span (since 12 years is about one Trojan orbital period). From the orbital elements we could calculate each object's heliocentric distance, geocentric distance, and phase angle over this 12-year interval. In combination with $H$, and assuming a linear phase law of $0.04 \mathrm{mag} \mathrm{deg}^{-1}$, we then calculated each object's $V$-band magnitude $m_{V}$ over this span. This range of dates was chosen since it falls within a period when the Spacewatch survey was surveying the sky down to $m_{V} \approx 21$ (Jedicke \& Metcalfe 1998; Larsen et al. 2007). In fact, Spacewatch either discovered outright or independently found almost all of the Trojans in our Spitzer sample.

We decided that a virtual Trojan in our simulation was considered "discovered" (and therefore available for inclusion in our Spitzer survey) if it ever became brighter than $m_{V}=20$ over the course of its orbit. This is a conservative choice in limiting magnitude; since ours is a simplistic model and does not explicitly take into account the actual sky coverage by the discovery surveys or the robustness of their ability to detect low signal-to-noise asteroids, we decided to pick a magnitude limit somewhat brighter than Spacewatch's nominal limit.

The result of the simulation is an ensemble of discovered, virtual objects that is a subset of the whole group of objects. We then created a plot of diameter versus albedo ( $D$ versus $\left.p_{R}\right)$ for these discovered objects that can be compared to the plot of real observations in Figure 1 . (The $R$-band albedo $p_{R}$ was calculated from $p$ by multiplying by 1.076 as in Section 4.1.) To do this comparison statistically, we followed the recipe for a two-dimensional Kolmogorov-Smirnov $(\mathrm{K}-\mathrm{S})$ test as described by Press et al. (1992).

To keep the model simple, we created the functional form of $P(p)$ as follows:

$P(p)= \begin{cases}0 & \text { if } p \leqslant 0, \\ C^{\prime} e^{-\left(p-p_{0}\right)^{2} / 2 \sigma_{p}^{2}}+C^{\prime \prime} \Pi\left(p_{l}, p_{l}+p_{w} ; p\right) & \text { if } p>0,\end{cases}$

where $\Pi$ is the boxcar function. In other words, the albedo distribution had a Gaussian, low-albedo component and a uniformly distributed, high-albedo component. Specifically, $p_{0}$ is the mean albedo of the low-albedo group; $\sigma_{p}$ is the standard deviation of the Gaussian; $p_{l}$ is the lower bound of the highalbedo group; and $p_{w}$ is the albedo width of the high-albedo group. (Note that for some parameter values some objects that belong to the ostensibly low-albedo Gaussian could have albedos that overlap with those from the high-albedo uniform distribution.) A fifth parameter, the fraction of objects with "high" albedo, $f_{h}$, controls the value $C^{\prime \prime}$ :

$$
f_{h}=\int_{p_{l}}^{p_{l}+p_{w}} C^{\prime \prime} \Pi\left(p_{l}, p_{l}+p_{w} ; p\right) d p=C^{\prime \prime} p_{w} .
$$

The overall normalization $\int_{-\infty}^{\infty} P(p) d p=1$ controls the relative scale of $C^{\prime}$ and $C^{\prime \prime}$, so this setup has five parameters to investigate. Note that we have not assumed any trend between diameter and albedo.

\footnotetext{
5 http://cfa-www.harvard.edu/iau/lists/JupiterTrojans.html
}

Our search through sample space is represented in Figure 5. Each panel shows a contour plot of the nominal probability that our observed plot of $D$ versus $p_{R}$ (Figure 1) and the simulated plot are drawn from the same two-dimensional distribution. As Press et al. (1992) describe, probabilities greater than about 0.2 may not be precise due to the simplistic nature of this formulation of the $\mathrm{K}-\mathrm{S}$ test, but still do indicate similar distributions.

The similarity of the contours in the panels of Figure 5 indicates that the "best" matches are consistently near $p_{0} \approx 0.07$ to 0.12 and $\sigma_{p} \approx 0.01$ to 0.06 . The fraction of high-albedo small Trojans $f_{h}$ is less constrained, but seems to be roughly under $30 \%$. The extent in albedo of that fraction is likewise not well constrained. This all depends somewhat on the a priori functional form of the distribution we have assigned, but the important and robust conclusion is that the small-Trojan albedo distribution is definitely not like that of the large Trojans $\left(p_{0} \approx 0.041, \sigma_{p} \approx 0.01, f_{h} \approx 3 \%\right.$; bottom half of Figure 3 ); the probabilities are far too small. This gives us confidence that the small Trojans really do have different surface properties even accounting for the discovery bias.

\subsection{Effect of Modeling Assumptions}

As stated in Section 3, the phase slope parameter $G$ influences the final results in Table 3. Re-running our thermal model for an assumed $G$ of 0.15 instead of 0.05 results in almost no change to the radii (at the $\sim 10 \mathrm{~m}$ level) and a reduction in the albedos by about $8 \%-9 \%$. Such a small change would not alter our conclusions.

More critical is the choice of $\eta$, since this certainly can have a significant effect on the calculated values of both $D$ and $p_{R}$. To gauge the influence that our assumptions have on our results, we re-analyzed our photometry in Table 2 using $\eta=1.25, \eta=1.6$, and $\eta=2.0$ instead of 0.94 . Each value would assume that the small Trojans had successively higher thermal inertia, similar to what has been measured in several near-Earth asteroids (e.g., Delbó et al. 2003). These three trial values of $\eta$ result in the diameters being (on average) $15 \%, 30 \%$, and $47 \%$ higher and the albedos being (on average) 24\%, 41\%, and 54\% lower than what we present in Table 3. Thus, if $\eta$ really were 2.0 then the albedos of the small Trojans would be sufficiently small so that the median value would approach that of the large Trojans, 0.050 versus 0.044 , and it would be less clear how significantly more reflective the small Trojans would be. However, this would mean that the thermal behavior of the small Trojans would be radically different from the large Trojans. In effect, an incorrect assumption of $\eta$ would not nullify the conclusion that the small Trojans are different from the large ones, it can only alter the way in which they are different.

But is it likely that smaller Trojans have higher thermal inertia due to having less regolith, or a large-grained regolith? The large Trojans $(D>140 \mathrm{~km})$ seem to have fine-grained silicates on their surfaces that produce mid-IR emission bands (Emery et al. 2006), indicating a fluffy regolith or a regolith where silicates are embedded in transparent grains. These concepts are also consistent with the average $\eta$ we found in Paper I. There is as yet no such detailed data on small Trojans (such as those in our current sample) to test whether the regolith properties change as a function of size.

Recent thermal studies of cometary nuclei are suggestive as a point of comparison, since the size matching between comets and small Trojans is reasonable, and since they are both classes of primitive objects. As mentioned earlier (Section 3.1), 


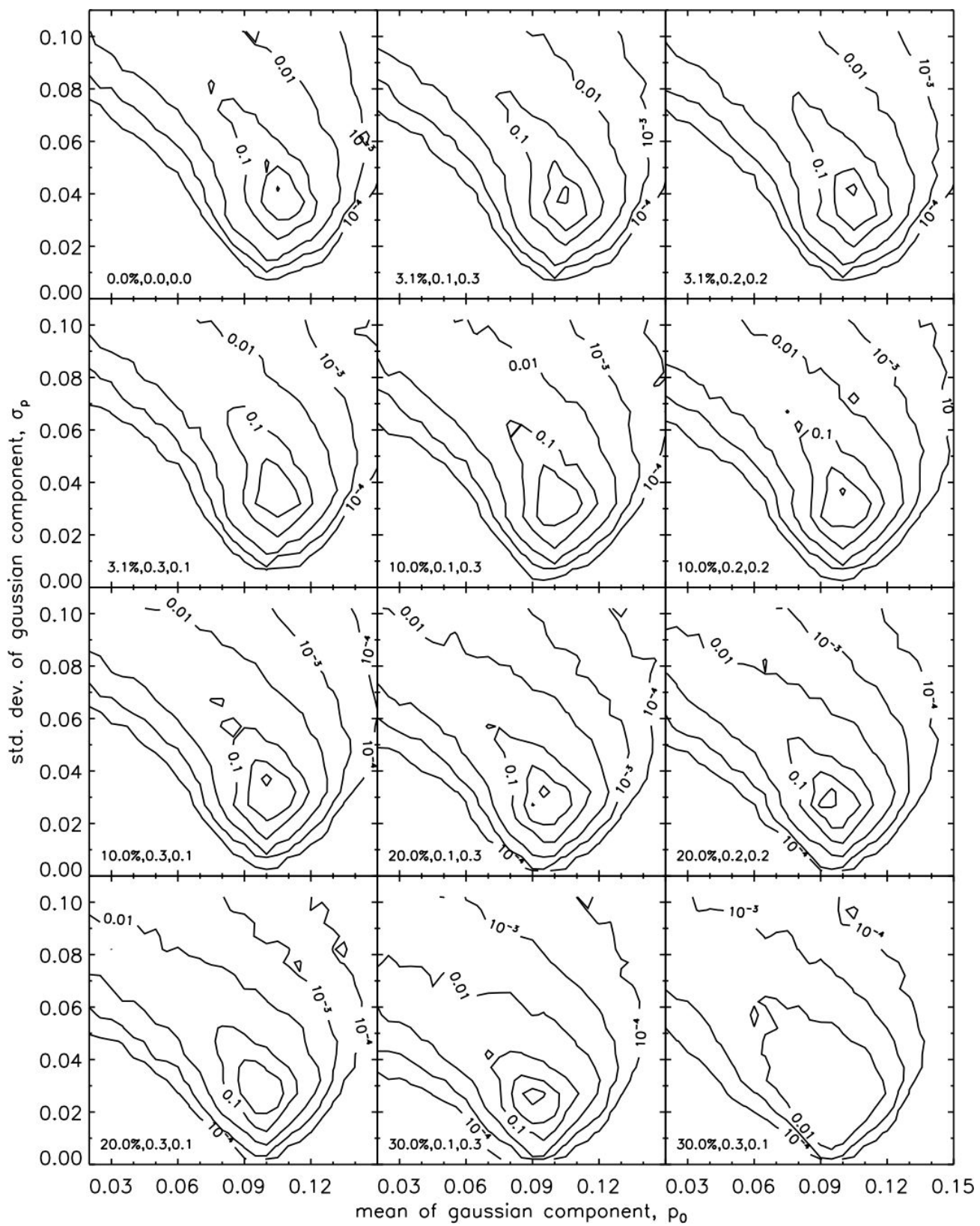

Figure 5. Contour plots of the probability that the observed $D$ vs. $p$ distribution seen in Figure 1 is drawn from the same distribution as that based on the simulations using the five-parameter model described in Section 4.2. Each panel represents different values of $f_{h}, p_{l}$, and $p_{w}$; the values are written in the lower left. Contours correspond to probabilities of $10^{-4}$ (outermost contour), $10^{-3}, 0.01,0.1,0.3$, and 0.5 .

work by many groups (e.g., Julian et al. 2000; Lamy et al. 2003; Groussin et al. 2004, 2007; Lisse et al. 2005) has shown that cometary nuclei have low thermal inertias, no more than approximately half that of the Moon and often consistent with zero. Furthermore, work by Fernández et al. (2008) has shown that the ensemble average of $\eta$ for about 50 cometary nuclei observed at 4-5 AU from the Sun is near unity. Such heliocentric distances are very near that of the Trojans. If the small Trojans are structurally similar to these comets, then an assumption of $\eta=0.94$ is quite reasonable.

\subsection{Comparison with Comets}

As noted above, the published properties of the Trojans are broadly compatible with those of the cometary nuclei. In particular, the Trojan optical color distribution resembles that of the cometary nuclei (Jewitt \& Luu 1990) in that both are deficient in ultrared material known to coat the surfaces of many Kuiper belt objects (Jewitt 2002). The albedos of the larger Trojans (Paper I) are likewise similar to the albedos of cometary nuclei, and suggest a carbonized, nonvolatile surface composition. Comparison between the physical properties of the comets and the Trojans is especially interesting in the context of the Nice model, in which Trojans and Jupiter-family comets are both products of the Kuiper belt (Morbidelli et al. 2005). The depletion of the ultrared matter on the comet nuclei and Trojans already argues either that this supposition is incorrect, or that the surface properties of Kuiper belt objects are modified after their removal from the Kuiper belt (Jewitt 2002). A systematic difference in the albedos would demand a similar interpretation.

The new results presented here tend to decrease the similarity with the comets, in the sense that when Trojans and nuclei of the same size are compared, the Trojan albedos are systematically 
higher. The strength of this statement is limited by the small sample of cometary nuclei for which reliable albedo determinations exist, something soon to be corrected by an ongoing survey of cometary nuclei (see Fernández et al. 2008).

\subsection{Size Distribution}

Previous workers derived the size distribution of the Trojans based on the magnitude distribution and an assumption of constant albedo. Jewitt et al. (2000) found a distribution consistent with two power laws; for the largest objects, with diameters $D \geqslant 70 \mathrm{~km}$, they found that the differential size distribution's power-law index is a relatively steep $q=5.5 \pm 0.9$, but for objects with $D$ between 4 and $40 \mathrm{~km}$, they measured $q=3.0 \pm 0.3$. The small end of the distribution was also measured by Yoshida \& Nakamura (2005) and Szabó et al. (2007), who found similar values for $q: 2.9 \pm 0.1$ and $3.2 \pm 0.25$, respectively. However, now we are in a position to make a better conversion between absolute magnitude $H$ and diameter $D$ since we have a relationship between $D$ and $p_{R}$ in Figures 1 and 2. The higher albedos found for small Trojans imply smaller diameters than expected, which would result in a flattening of the size distribution relative to the constant albedo case.

To quantify this effect, we represent the $D$ versus $p_{R}$ trend in Figure 2 by an ad hoc function. We used the data in Figure 2 to fit (by least-squares) the coefficients to the following fourth-order polynomial:

$$
p_{R}(D)=\sum_{m=0}^{4} c_{m} x^{m}
$$

where $x=\log (D / 1 \mathrm{~km})$. However, for $D<5 \mathrm{~km}$, we capped $p_{R}$ at 0.3 , and for $D>143 \mathrm{~km}$ we set a floor of $p_{R}=0.044$. The coefficients are

$$
\begin{aligned}
& c_{0}=1.540 ; c_{1}=-3.094 ; c_{2}=2.443 ; c_{3}=-0.858 \\
& \text { and } c_{4}=0.112 .
\end{aligned}
$$

This function is plotted with the data in Figure 2.

We converted the differential size distribution provided by Jewitt et al. (2000) for the L4 swarm-their Equations (8) and (9)—back to a luminosity function, i.e., a function of $H$, using their 0.04 assumed albedo. Using our Equation (3), we could convert $H$ to a more robust estimate of $D$ and thus then derive a new size distribution.

The result is shown in Figure 6. The kink in our size distribution near $D=5 \mathrm{~km}$ is due to the break in our $p_{R}(D)$ function at that diameter. The curvature to the middle segment between $D=5 \mathrm{~km}$ and $D=35 \mathrm{~km}$ is due to the curvature in $p_{R}(D)$ at those diameters, but in log-log space the segment approximates a power law.

The other implication of Figure 6 is that the number of Trojans larger than a given size is lower than previously estimated (assuming that there is no trend of beaming parameter with diameter). Figure 6 indicates that there are approximately $9 \times 10^{4}$ Trojans in the L4 swarm with diameter larger than $2 \mathrm{~km}$, and about $3 \times 10^{5} \mathrm{~L} 4$ Trojans with diameter larger than $1 \mathrm{~km}$. This is about a factor of 2 smaller than the estimate obtained by Jewitt et al. (2000) using a constant albedo and $q=3$. Other estimates of the Trojan population (e.g., Yoshida \& Nakamura 2005; Szabó et al. 2007; Nakamura \& Yoshida 2008) that assume a constant albedo and use a similar magnitude distribution would have similar downward corrections to the population estimate.

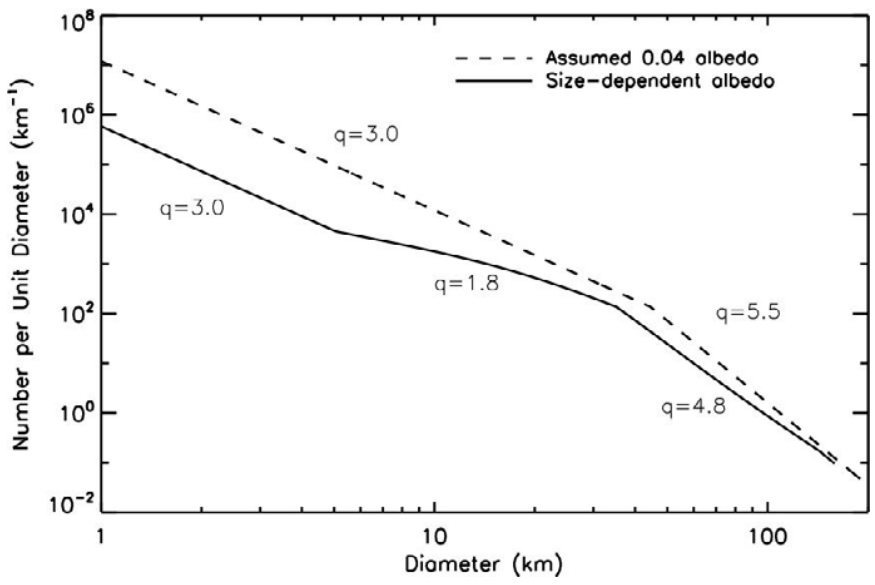

Figure 6. Differential size distribution of Trojans. Dashed line is the distribution derived by Jewitt et al. (2000) based on an assumed albedo of 0.04 that was size-independent. Solid line is our new derivation based on their survey data and the size-dependent albedo shown in Figure 2. The equivalent power-law slopes, $q$, of each segment in both distributions are shown.

\subsection{Origin}

The observed albedo versus diameter relation could have a number of causes, ranging from the profound to the insignificant. The degree of heating experienced by a solid body due to the decay of embedded radioactive nuclei increases with the diameter, all else being equal. One hundred kilometer scale Trojans will experience a temperature increase from trapped radio-nuclei approximately 10 times larger than will Trojans only $10 \mathrm{~km}$ in scale. Thus, it is tempting to think that the observed albedo versus size relation might be an artifact of different degrees of metamorphism in the Trojans, assuming that these objects trapped sufficient quantities of short-lived radionuclei such as ${ }^{26} \mathrm{Al}$ and ${ }^{60} \mathrm{Fe}$ to be appreciably heated. Arguing against this possibility is the size distribution of the Trojans, which resembles (at least) two power laws intersecting at about 30-40 km diameter (i.e., neatly separating the sample in the present study from that in Paper I). Trojans larger than this are thought to be survivors of a primordial population while those smaller than this are more likely to be products of past, shattering collisions. If so, the small objects in the present sample were once part of larger bodies that must have been radioactively heated, and no simple difference based on the efficiency with which radiogenic heat can be trapped is expected.

The albedo versus diameter relation may instead suggest the action of some process involving collisions. The collisional lifetimes of small Trojans are short compared to the larger objects. If the exposed surfaces of Trojan asteroids are progressively darkened, for example by the irradiation and dehydrogenization of hydrocarbons (e.g., Thompson et al. 1987; Moroz et al. 2004), then it is at least qualitatively reasonable to expect an albedo versus diameter trend with the sense observed.

Such a cause might also imply that there should be a colordiameter trend in the Trojans, since irradiation can change the reflectance slope as well as the albedo. Laboratory results indicate that the changes in the slope depend on dosages and on the original make-up of the surface (e.g., Moroz et al. 2003, 2004), so there may be no easy answer as to what colors to expect on Trojans that have suffered various amounts of weathering. Observationally, Jewitt \& Luu (1990) found a trend where smaller Trojans (i.e., Trojans with surfaces that are statistically younger) have redder surfaces, and this trend was corroborated 
for D-type asteroids by Fitzsimmons et al. (1994), by Lagerkvist et al. (2005) (for Cybeles), and by Dahlgren et al. (1997) (for Hildas). Recent work on a wider sample of Trojans has let some workers study colors of "background" Trojans as distinct from those of Trojans in dynamical families. In particular, Fornasier et al. (2007) conclude that there is no statistically significant trend between color and size, while Roig et al. (2008) argue that among the background population, it is the larger Trojans that are redder. In short, the observational situation regarding a color versus diameter relation currently remains unresolved. Future visible and near-IR data sets on a larger number of familial and nonfamilial Trojans and on Trojans down to small sizes may shed more light on this issue.

In any case, in a scenario where collisions play a significant role in determining the sizes of the small Trojans that now exist in the swarms, one might expect the size distribution power law to more closely mimic the Dohnanyi power law for collisional fragments. Figure 6 indicates however that we have now moved the small-size power law to a shallower slope, away from the collisional equilibrium value. So while collisions likely are influencing the distribution, there is as yet no simple explanation for Figure 6.

\section{SUMMARY}

We have measured the $24 \mu \mathrm{m}$ thermal emission from 44 small Jovian Trojans using the Spitzer Space Telescope and the $R$-band reflected sunlight from 32 of those to derive effective diameters and albedos. Our sample covers diameters from 5 to $24 \mathrm{~km}$, significantly smaller than the large Trojans we sampled in an earlier survey ( $D>57 \mathrm{~km}$; Paper I). We reach the following conclusions.

1. The measured mean $R$-band geometric albedo of the small Trojans in our sample is $0.121 \pm 0.003$, and the median is $0.105 \pm 0.004$. Including only objects for which we have multiwavelength data, the mean is $0.137 \pm 0.004$, and the median is $0.117 \pm 0.005$. These are significantly higher values than the $0.044 \pm 0.001$ found for the large Trojans (Paper I).

2. The spread in $R$-band albedos among the small Trojans exceeds that of the large Trojans, with a standard deviation of about 0.065 versus 0.008 (Paper I).

3 . The $R$-band geometric albedo decreases with increasing diameter in the 5-24 km range. This correlation is significant at the $3.4 \sigma$ level, and becomes more significant $(6.8 \sigma)$ when we include the large Trojans from our earlier work (Paper I).

4. The differences in albedo distribution between the large and small Trojans are unlikely to be caused by either (a) the nonsimultaneity in our optical/thermal data, or (b) a discovery bias toward finding Trojans of high albedo in the first place. It is possible that the albedo differences are artifacts of using a size-independent IR beaming parameter in interpreting the radiometry (and that the small Trojans have a different ensemble average thermal inertia than the large ones do), but we believe this possibility to be unlikely.

5. The origin of the albedo-diameter relation is unknown but collisions, which shatter and create small bodies on much shorter timescales than large bodies, may be implicated.

6. The measured size dependence of the albedo tends to flatten the best-fit power-law size distribution index relative to the value computed under the assumption of constant albedo. We find that the differential power-law index that best matches published survey data for objects in the $5 \leqslant D \leqslant$ $30 \mathrm{~km}$ range is $q \approx 1.8$, whereas the value under the constant albedo assumption is $q \approx 3.0$.

7. This flattened size distribution implies that there are about a factor of 2 fewer objects of radius greater than $1 \mathrm{~km}$ than estimated when assuming a 0.04 constant albedo. For example, using the magnitude distribution reported by Jewitt et al. (2000), we find that there are about $9 \times 10^{4}$ L4 Trojans with radius greater than $1 \mathrm{~km}$ instead of the $1.6 \times 10^{5}$ inferred with the constant albedo assumption.

We thank John Dvorak for operating the UH telescope, and for the helpful comments of the anonymous referee and of Rachel Stevenson. This work is based in part on observations made with the Spitzer Space Telescope, which is operated by the Jet Propulsion Laboratory, California Institute of Technology, under a contract with NASA. Support for this work was provided by NASA through an award issued by JPL/Caltech to Y.R.F. and D.J., and also through Planetary Astronomy grant NNG06GG08G to D.J. We acknowledge the reference material provided by the MPC.

Facilities: Spitzer (), UH:2.2m ()

\section{REFERENCES}

Allen, D. A. 1970, Nature, 227, 158

Cruikshank, D. P., Dalle Ore, C. M., Roush, T. L., Geballe, T. R., Owen, T. C., de Bergh, C., Cash, M. D., \& Hartmann, W. K. 2001, Icarus, 153, 348

Dahlgren, M., Lagerkvist, C. I., Fitzsimmons, A., Williams, I. P., \& Gordon, M. 1997, A\&A, 323, 606

Delbó, M., Dell'Oro, A., Harris, A. W., Mottola, S., \& Mueller, M. 2007, Icarus, 190,236

Delbó, M., Harris, A. W., Binzel, R. P., Pravec, P., \& Davies, J. K. 2003, Icarus, 166,116

Dotto, E., Emery, J., Barucci, M., Morbidelli, A., \& Cruikshank, D. 2008, in The Solar System Beyond Neptune, ed. M. A. Barucci et al. (Tucson, AZ: Univ. Arizona Press), 383

Dumas, C., Owen, T., \& Barucci, M. A. 1998, Icarus, 133, 221

Emery, J. P., \& Brown, R. H. 2003, Icarus, 164, 104

Emery, J. P., Cruikshank, D. P., \& van Cleve, J. 2006, Icarus, 182, 496

Fernández, Y. R., Campins, H., Kassis, M., Hergenrother, C. W., Binzel, R. P., Licandro, J., Hora, J. L., \& Adams, J. D. 2006, AJ, 132, 1354

Fernández, Y. R., Jewitt, D. C., \& Sheppard, S. S. 2003, AJ, 126, 1563

Fernández, Y. R., et al. 2008, in Asteroids, Comets, Meteors 2008, LPI Contrib. 1405, (Houston, TX: Lunar and Planetary Institute), 8307

Fitzsimmons, A., Dahlgren, M., Lagerkvist, C. I., Magnusson, P., \& Williams, I. P. 1994, A\&A, 282, 634

Fornasier, S., Dotto, E., Hainaut, O., Marzari, F., Boehnhardt, H., de Luise, F., \& Barucci, M. A. 2007, Icarus, 190, 622

Garaud, P., \& Lin, D. N. C. 2007, ApJ, 654, 606

Gordon, K. D., et al. 2005, PASP, 117, 503

Groussin, O., Lamy, P., \& Jorda, L. 2004, A\&A, 413, 1163

Groussin, O., et al. 2007, Icarus, 187, 16

Groussin, O., et al. 2009, Icarus, 199, 568

Harris, A. W. 1998, Icarus, 131, 291

Harris, A. W., \& Davies, J. K. 1999, Icarus, 142, 464

Jedicke, R., \& Metcalfe, T. S. 1998, Icarus, 131, 245

Jewitt, D. C. 2002, AJ, 123, 1039

Jewitt, D. C., \& Luu, J. X. 1990, AJ, 100, 933

Jewitt, D. C., Sheppard, S., \& Porco, C. 2004, in Jupiter: The Planet, Satellites and Magnetosphere, ed. F. Bagenal (Cambridge: Cambridge Univ. Press), 263

Jewitt, D. C., Trujillo, C. A., \& Luu, J. X. 2000, AJ, 120, 1140

Julian, W. H., Samarasinha, N. H., \& Belton, M. J. S. 2000, Icarus, 144, 160

Lagerkvist, C. I., Moroz, L., Nathues, A., Erikson, A., Lahulla, F., Karlsson, O., \& Dahlgren, M. 2005, A\&A, 432, 349

Lamy, P., Biesecker, D. A., \& Groussin, O. 2003, Icarus, 163, 142

Lamy, P. L., Toth, I., Fernández, Y. R., \& Weaver, H. A. 2004, in Comets II, ed. M. C. Festou et al. (Tucson, AZ: Univ. Arizona Press), 223

Lamy, P. L., Toth, I., Groussin, O., Jorda, L., Kelley, M. S., \& Stansberry, J. A. 2008, A\&A, 489, 777 
Landolt, A. U. 1992, AJ, 104, 340

Larsen, J. A., et al. 2007, AJ, 133, 1247

Lebofsky, L. A., \& Spencer, J. S. 1989, in Asteroids II, ed. R. P. Binzel et al. (Tucson, AZ: Univ. Arizona Press), 128

Li, J.-Y., A'Hearn, M. F., McFadden, L. A., \& Belton, M. J. S. 2007, Icarus, 188,195

Lisse, C. M., et al. 2005, ApJ, 625, L139

Luu, J., Jewitt, D., \& Cloutis, E. 1994, Icarus, 109, 133

Makovoz, D., \& Marleau, F. R. 2005, PASP, 117, 1113

Mann, R. K., Jewitt, D., \& Lacerda, P. 2007, AJ, 134, 1133

Marzari, F., \& Scholl, H. 2007, MNRAS, 380, 479

Marzari, F., Scholl, H., Murray, C., \& Lagerkvist, C. 2002, in Asteroids III, ed. W. F. Bottke et al. (Tucson, AZ: Univ. Arizona Press), 725

Matson, D. K. 1971, PhD thesis, California Institute of Technology

Morbidelli, A., Levison, H. F., Tsiganis, K., \& Gomes, R. 2005, Nature, 435, 462

Moroz, L. V., Baratta, G., Distefano, E., Strazzulla, G., Starukhina, L. V., Dotto, E., \& Barucci, M. A. 2003, EM\&P, 92, 279

Moroz, L., Baratta, G., Strazzula, G., Starukhina, L., Dotto, E., Barucci, M. A., Arnold, G., \& Distefano, E. 2004, Icarus, 170, 214
Morrison, D. 1973, Icarus, 19, 1

Nakamura, T., \& Yoshida, F. 2008, PASJ, 60, 293

Pravec, P., Harris, A. W., \& Michalowski, T. 2002, in Asteroids III, ed. W. F. Bottke et al. (Tucson, AZ: Univ. Arizona Press), 113

Press, W. H., Teukolsky, S. A., Vetterling, W. T., \& Flannery, B. P. 1992, Numerical Recipes in Fortran (2nd ed.; New York: Cambridge Univ. Press) Rieke, G. H., et al. 2004, ApJS, 154, 25

Roig, F., Ribeiro, A. O., \& Gil-Hutton, R. 2008, A\&A, 483, 911

Spencer, J. R., Lebofsky, L. A., \& Sykes, M. V. 1989, Icarus, 78, 337

Spitzer Science Center 2008, Spitzer Space Telescope Multiband Imaging Photometry for Spitzer (MIPS) Data Handbook, version 3.3.1

Stansberry, J., Grundy, W., Brown, M., Cruikshank, D., Spencer, J., Trilling, D., \& Margot, J.-L. 2008, in The Solar System Beyond Neptune, ed. M. A Barucci et al. (Tucson, AZ: Univ. Arizona Press), 161

Szabó, G. M., Ivezić, Ž., Jurić, M., \& Lupton, R. 2007, MNRAS, 377, 1393

Thompson, W., Murray, B. G. J. P. T., Khare, B. N., \& Sagan, C. 1987, J. Geophys. Res., 92, 14933

Werner, M., et al. 2004, ApJS, 154, 1

Yang, B., \& Jewitt, D. 2007, AJ, 134, 223

Yoshida, F., \& Nakamura, T. 2005, AJ, 130, 2900 